\title{
Système de tarif lié à la prestation pour la psychiatrie hospitalière
}

\author{
Beatrix Meyera, Bettina Holzer \\ ${ }^{a}$ Cheffe de la division Tarifs et économie de la santé pour les médecins hospitaliers \\ ${ }^{b}$ Dr méd., division Tarifs et économie de la santé pour les médecins hospitaliers
}

\begin{abstract}
La version préliminaire du système tarifaire TARPSY 0.2 présente différentes variantes qui conjuguent forfaits journaliers et forfaits par cas. La FMH privilégie une structure tarifaire qui repose exclusivement sur des forfaits journaliers liés à la prestation et rejette catégoriquement tout élément de forfait par cas.
\end{abstract}

Aujourd'hui, les traitements psychiatriques hospitaliers sont indemnisés par des forfaits journaliers, dont la forme varie d'un canton et d'un partenaire contractuel à l'autre. Désormais, le législateur exige un système de rémunération lié à la prestation uniforme dans toute la Suisse pour la psychiatrie hospitalière. Ce système, en cours d'élaboration sous l'égide de SwissDRG SA, vise à créer une nouvelle structure tarifaire simple et adaptée présentant le moins possible d'incitatifs erronés. La Haute école zurichoise des sciences appliquées (ZHAW), chargée de la développer, a d'ores et déjà élaboré une version préliminaire de TARPSY 0.2 en se concentrant principalement sur la psychiatrie de l'adulte. En ce qui concerne la pédopsychiatrie, les travaux sont nettement moins avancés en raison notamment des données insuffisantes à disposition. L'introduction de TARPSY est prévue pour 2018.

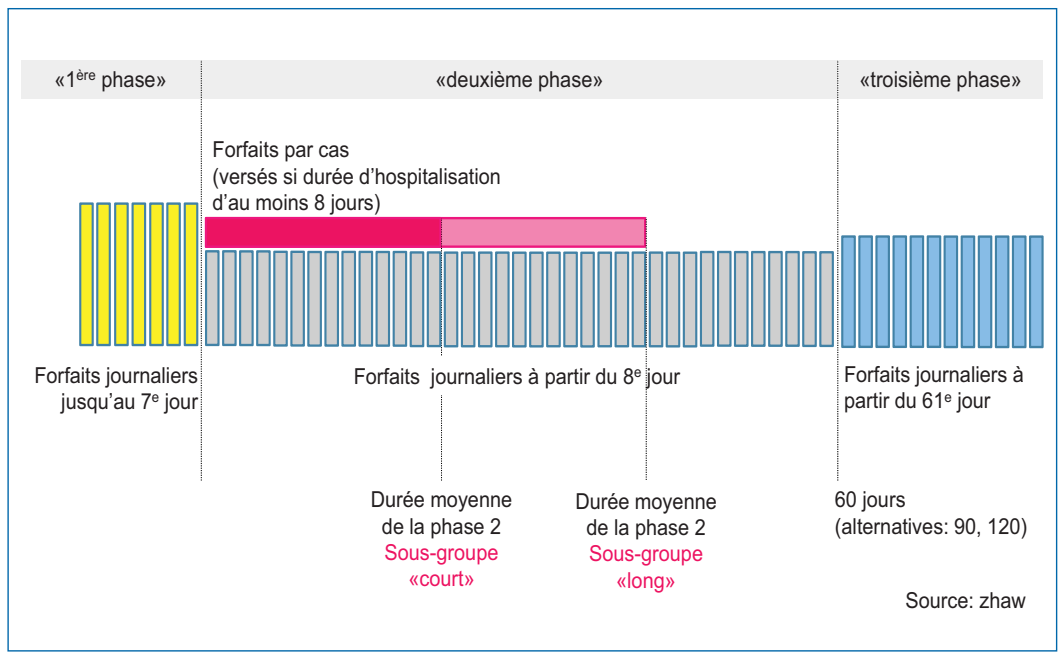

Figure 1: Modèle tarifaire en trois phases pour la psychiatrie hospitalière de l'adulte.

\section{Un modèle en trois phases combinant forfaits journaliers et forfaits par cas}

Les hospitalisations en psychiatrie de l'adulte seront réparties en trois phases (fig. 1). La première, de sept jours, est rémunérée par des forfaits journaliers. La deuxième commence au huitième jour, et se compose d'une conjonction de forfaits journaliers et de forfaits par cas, suivie de la troisième phase pour les patients de longue durée avec uniquement des forfaits journaliers. Actuellement, une analyse des données est en cours pour évaluer si cette dernière phase doit commencer au $61^{\mathrm{e}}, 91^{\mathrm{e}}$ ou $121^{\mathrm{e}}$ jour.

Les séjours psychiatriques hospitaliers sont classés par groupes de coûts psychiatriques (PCG - Psychiatric cost groups) les plus homogènes possible, en fonction du diagnostic principal et de l'intensité du traitement. L'affectation à un des neuf [1] groupes de diagnostic principal en psychiatrie se fait sur la base de la CIM 10 GM [2]. La ZHAW propose l'HoNOS [3] pour mesurer l'intensité des traitements psychiatriques de l'adulte.

S'agissant des forfaits journaliers de la phase 1 et 2, HoNOS permet de distinguer entre traitement ordinaire et traitement intensif dans chacun des groupes de diagnostic psychiatrique. Dans le cas des forfaits par cas en phase 2, HoNOS permet en plus de constituer des groupes de patients en fonction de la durée plus ou moins longue de l'hospitalisation.

Dans ce contexte, les phases 1 et 2 [4] font chacune état de 18 PCG pour les forfaits journaliers, auxquels viennent s'ajouter 36 PCG pour les forfaits par cas de la phase 2 [5]. L'intensité du traitement n'est quant à elle plus prise en compte dans la phase 3 , divisée en seulement trois PCG. Enfin, le tarif pour les séjours hospitaliers est calculé d'après le coût relatif du PCG, le nombre 
de jours d'hospitalisation et le tarif de base négocié par les partenaires tarifaires [6].

\section{Forfaits par cas inadaptés à la psychiatrie}

Pour la phase 2, la ZHAW a proposé plusieurs variantes avec des parts différentes de forfaits par cas de $0 \%, 25 \%$, $50 \%, 75 \%$ et $100 \%$, et relevé que plus la proportion diminue, plus la qualité des prévisions [7] s'améliore; en l'occurrence, les meilleurs résultats sont obtenus avec la variante à $0 \%$ de forfaits par cas, soit $100 \%$ de forfaits journaliers. La ZHAW signale à cet égard qu'une part de forfaits par cas incite à raccourcir la durée de l'hospitalisation. Cette part ne peut cependant pas être fixée en fonction des données disponibles mais doit être définie par les partenaires de SwissDRG SA.

Comme la mise en place d'une part de forfaits par cas au terme de la phase 1 risque d'engendrer des variations dans les revenus, SwissDRG SA souhaite examiner comment combiner des éléments du modèle TARPSY avec ceux du modèle SwissDRG afin d'éviter de telles variations.

\section{La FMH rejette catégoriquement tout élément de forfait par cas dans la structure tarifaire et privilégie des forfaits journaliers liés à la prestation.}

La FMH plaide pour une structure tarifaire entièrement composée de forfaits journaliers et rejette catégoriquement les variantes incluant une part de forfaits par cas car la durée d'hospitalisation pour un même diagnostic ne dépend pas uniquement du médecin ou de l'institution psychiatrique mais aussi du patient et de son environnement social. De plus, le corps médical n'a souvent aucune influence sur les placements à des fins d'assistance (PAFA) qui sont gérés différemment d'un canton à l'autre et qui, de ce fait, n'ont pas la même proportion en fonction du canton et de la clinique. Rappelons que les internements forcés en Suisse représentent en moyenne un quart de tous les internements [8]. Par ailleurs, la FMH redoute qu'inclure une part de forfaits par cas dans la rémunération crée une pression économique incitant à anticiper les sorties d'hôpital sans justification médicale, ce qui se reporterait ensuite sur une hausse des réhospitalisations.

\section{Analyse en cours d'alternatives à HoNOS}

Pour la FMH, HoNOS n'est pas pertinent pour déterminer l'intensité d'un traitement en psychiatrie de l'adulte. Jusqu'à présent, cette classification a été utilisée et testée pour mesurer la qualité mais pas pour développer la structure tarifaire. Par ailleurs, la saisie dans HoNOS n'est pas à l'abri de manipulations. Selon la ZHAW, le BSCL [9] ne s'est pas non plus révélé être un bon instrument de contrôle et ne sera donc pas réutilisé mais, pour l'heure, aucune alternative n'existe. De plus, le corps médical redoute une hausse de la charge administrative car la collecte des données HoNOS demande davantage de temps pour TARPSY que pour l'ANQ [10]. Des problèmes similaires se présentent avec l'HoNOSCA [11] qui devrait servir à mesurer l'intensité des traitements en psychiatrie de l'enfant et de l'adolescent.

Par conséquent, la FMH exige que d'autres critères soient évalués pour déterminer l'intensité des traitements, tels que les placements à des fins d'assistance (PAFA). Entretemps, la ZHAW a annoncé que pour la version TARPSY 0.3, elle examinerait des variables supplémentaires, autres qu'HoNOS, comme les PAFA ou l'âge. Il est par ailleurs réjouissant que le schéma de classification multiaxial (SCM) soit examiné pour la psychiatrie de l'enfant et de l'adolescent car c'est une exigence que la FMH a exprimé dans son papier de position [12] sur TARPSY.

\section{Une meilleure qualité des données est primordiale}

Le développement de TARPSY 0.2 pour la psychiatrie de l'adulte repose sur les données de l'année 2013 de 17 cliniques psychiatriques. Dans l'ensemble, faute de qualité suffisante des données, seul un tiers des cas saisis ont pu être utilisés, ce qui correspond environ à un cinquième des cas hospitaliers annuels. Pour améliorer la qualité des données concernant les PAFA, il est nécessaire de faire une distinction nette entre les variables statistiques "volontaire» et "privation de liberté à des fins d'assistance» (prédécesseur du PAFA) car la saisie de ces variables ne se fait actuellement pas de manière uniforme. En psychiatrie de l'enfant et de l'adolescent, seules quatre cliniques ont livré leurs données, deux d'entre elles ayant utilisé le SCM. Pour continuer le développement de TARPSY, il est primordial que toutes les cliniques psychiatriques mettent des données de bonne qualité à disposition.

\section{Importance des cliniques de jour et des cliniques de nuit}

Il est regrettable que les cliniques de jour et les cliniques de nuit n'aient pas été prises en compte dans TARPSY 0.2. Pour la FMH, elles doivent être incluses dans le système tarifaire TARPSY car leur sous-rémunération actuelle incite à hospitaliser les patients non 
pas pour des raisons médicales mais pour un motif financier. Cela va à l'encontre du bien-être des patients et renchérit inutilement le système de santé. Il est important que les patients puissent toujours bénéficier du traitement le mieux adapté, indépendamment du fait qu'ils doivent se rendre à l'hôpital ou dans une clinique de jour / de nuit ou encore suivre le traitement en ambulatoire.

\section{Réglementation transitoire et recherche concomitante nécessaires}

Comme la structure tarifaire TARPSY est entièrement nouvelle, la FMH estime qu'il est indispensable de mettre en place une réglementation transitoire de plusieurs années pour éviter les effets négatifs pendant la phase d'introduction. De plus, une recherche concomitante doit être lancée avant l'introduction de TARPSY

\section{En psychiatrie, la durée de traitement dépend de l'environnement social du patient.}

car c'est le seul moyen d'identifier suffisamment tôt les éventuelles distorsions de la nouvelle structure tarifaire et d'introduire les contre-mesures nécessaires.

\section{Conclusion et perspective}

La FMH recommande de développer le système tarifaire TARPSY exclusivement avec des forfaits journaliers liés à la prestation et de renoncer entièrement aux forfaits par cas.

Pour la FMH, les possibilités de manipuler la saisie dans HoNOS et HoNOSCA disqualifient ces deux échelles de classification pour déterminer l'intensité de traitement. Par conséquent, la FMH se félicite que la ZHAW examine d'autres alternatives pour TARPSY 0.3 comme les placements à des fins d'assistance pour la psychiatrie de l'adulte et le schéma de classification multiaxial pour la psychiatrie de l'enfant et de l'adolescent. Enfin, la FMH rappelle l'importance que revêt la qualité des données livrées par les cliniques pour le développement de la structure tarifaire. Conjointement avec ses sociétés de discipline, elle poursuit activement ses efforts pour élaborer une structure tarifaire liée à la prestation.

1 Un dixième groupe de diagnostic principal a fait l'objet d'une analyse pour les diagnostics principaux non psychiatriques.

2 CIM 10 GM: classification statistique internationale des maladies et des problèmes de santé connexes, $10^{\mathrm{e}}$ révision, German Modification, sert de base de codage des diagnostics pour les statistiques médicales en Suisse.

3 HoNOS: Health of the Nation Outcome Scales, instrument d'évaluation externe.

4 Un PCG supplémentaire vient s'ajouter au cas où le dixième groupe de diagnostic principal est utilisé pour les diagnostics principaux non psychiatriques.

5 Idem 4

6 Le tarif en CHF se calcule de la manière suivante: tarif de base en $\mathrm{CHF} \times\left[\left(\right.\right.$ forfait journalier $_{1} \times$ durée d'hospitalisation $\left.{ }_{1}\right)+$ forfait par cas $+\left(\right.$ forfait journalier $_{2} \times$ durée d'hospitalisation $\left.\left.{ }_{2}\right)\right]+($ forfait journalier $_{3} \times$ durée d'hospitalisation $\left.{ }_{3}\right)$ ].

7 Le calcul de l'écart moyen entre les coûts d'un cas et les coûts concrets détermine la qualité de prévision des modèles.

8 Analyse 2011 des fichiers 2009 des données supplémentaires en psychiatrie par l'Obsan, cf. Gassmann, Jürg, 2011. Wirksamkeit des Rechtsschutzes bei psychiatrischen Zwangseinweisungen in der Schweiz (en allemand avec résumé en français). Etude établie à la demande de l'Office fédéral de la santé publique. www.bag.admin.ch

9 BSCL: Brief Symptom Checklist, instrument d'autoévaluation

10 ANQ: Association nationale pour le développement de la qualité dans les hôpitaux et les cliniques.

11 HoNOSCA: Health of the Nation Outcome Scales for Children and Adolescents.

12 Papier de position de la FMH concernant le système tarifaire de la psychiatrie (TARPSY), 2013, www.fmh.ch $\rightarrow$ Tarifs hospitaliers $\rightarrow$ Positions $\rightarrow$ Papiers de position. 